\title{
Correlation between Fissured Fibrous Cap and Contrast Enhancement: Preliminary Results with the Use of CTA and Histologic Validation
}

\author{
L. Saba, E. Tamponi, E. Raz, L. Lai, R. Montisci, M. Piga, and G. Faa
}

\begin{abstract}
BACKGROUND AND PURPOSE: Previous studies demonstrated that carotid plaques analyzed by CTA can show contrast plaque enhancement. The purpose of this preliminary work was to evaluate the possible association between the fissured fibrous cap and contrast plaque enhancement.
\end{abstract}

MATERIALS AND METHODS: Forty-seven consecutive ( $m e n=25$; average age $=66.8 \pm 9$ years) symptomatic patients studied by use of a multidetector row CT scanner were prospectively analyzed. CTA was performed before and after contrast and radiation doses were recorded; analysis of contrast plaque enhancement was performed. Patients underwent carotid endarterectomy en bloc; histologic sections were prepared and evaluated for fissured fibrous cap and microvessel attenuation. The Mann-Whitney test was performed to evaluate the differences between the 2 groups. A multiple logistic regression analysis was performed to assess the effect of fissured fibrous cap and microvessel attenuation on contrast plaque enhancement. Receiver operating characteristic curve and area under the curve were also calculated.

RESULTS: Twelve patients had fissured fibrous cap. In 92\% (11/12) of fissured fibrous cap-positive plaques, we found contrast plaque enhancement, whereas in $69 \%$ (24/35) of the plaques without fissured fibrous cap contrast plaque enhancement was found. The MannWhitney test showed a statistically significant difference between the contrast enhancement in plaques with fissured fibrous cap (Hounsfield units $=22.6)$ and without fissured fibrous cap (Hounsfield units $=12.9)(P=.011)$. On the regression analysis, both fissured fibrous cap and neovascularization were associated with contrast plaque enhancement $(P=.0366$ and $P=.0001)$. The receiver operating characteristic curve confirmed an association between fissured fibrous cap and contrast plaque enhancement with an area under the curve of $0.749(P=.005)$.

CONCLUSIONS: The presence of fissured fibrous cap is associated with contrast plaque enhancement. Histologic analysis showed that the presence of fissured fibrous cap is associated with a larger contrast plaque enhancement compared with the contrast plaque enhancement of plaques without fissured fibrous cap.

ABBREVIATIONS: $\mathrm{CEA}=$ carotid endarterectomy; $\mathrm{CPE}=$ carotid plaque enhancement; $\mathrm{CTDI}=\mathrm{CT}$ dose index; $\mathrm{DLP}=$ dose-length product; $\mathrm{FFC}=$ fissured fibrous cap; $\mathrm{ROC}=$ receiver operating characteristic; $\mathrm{HU}=$ Hounsfield Units

$\mathbf{T}$ he presence of fissured fibrous cap (FFC) in the carotid artery plaque is associated with an increased risk of cerebrovascular events, and therefore FFC is considered one element that makes a carotid plaque "vulnerable." ${ }^{1-3}$ Identification of this condition is important to obtain a better stratification of stroke risk. $^{1}$

Received June 15, 2013; accepted after revision July 30

From the Departments of Radiology (L.S., M.P.), Pathology (E.T., L.L., G.F.), and Vascular Surgery (R.M.), Azienda Ospedaliero Universitaria, Monserrato (Cagliari), Italy; Department of Radiology (E.R.), New York University School of Medicine, New York, New York; and Department of Neurology and Psychiatry (E.R.), Sapienza University of Rome, Italy.

Please address correspondence to Luca Saba, MD, Department of Radiology, Azienda Ospedaliero Universitaria (A.O.U.), di Cagliari-Polo di Monserrato s.s. 554 Monserrato (Cagliari) 09045, Italy; e-mail: lucasaba@tiscali.it

http://dx.doi.org/10.3174/ajnr.A3759
The FFC has been studied by use of MR imaging, demonstrating the potential of MR imaging to detect the rupture of the fibrous cap, ${ }^{4,5}$ with or without the use of gadolinium. ${ }^{6,7}$ Recently, with the use of CTA and morphologic analysis of the carotid plaque, the FFC was documented. ${ }^{8}$ Even though the rupture of FFC has been shown to be associated with enhancement on MR imaging, ${ }^{7}$ this association has not yet been demonstrated by use of CT. The carotid plaque enhancement (CPE) on CTA is associated with plaque instability ${ }^{9-11}$; CPE is associated with microvessel attenuation, but the neovascularization alone cannot be the only factor because some plaques with high $\mathrm{CPE}$ do not show neovascularization. ${ }^{9}$

Our hypothesis is that the rupture of the FFC is an independent factor related to the $\mathrm{CPE}$, and we aim to evaluate this association. 

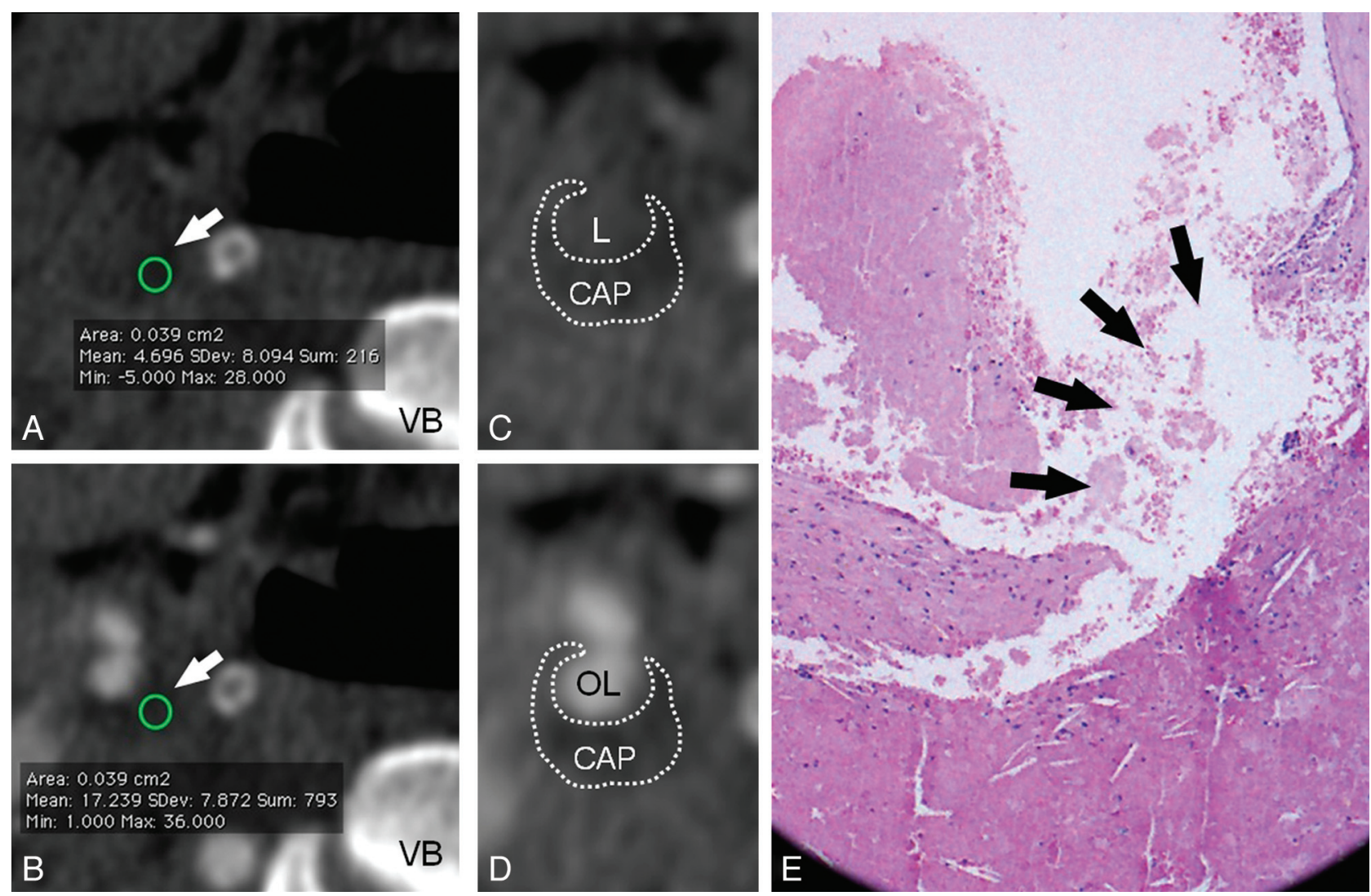

FIG 1. A 71-year-old male patient. In CT analysis, before $(A)$ and after (B) contrast, the carotid artery plaque is visible (white arrows) and the CPE $\mathrm{HU}$ value is shown. The circles, indicated by the white arrows, represent the area of the plaque that was assessed (in this case, the area was 0.039 $\mathrm{cm}^{2}$ ); in the basal scan, the average $\mathrm{HU}$ value was 4.696, whereas after administration of contrast material it was 17.239 (VB indicates vertebral body). In $C$ and $D$, the same images as $A$ and $B$ are shown with a zoom factor of $150 \%$ and demonstrate the contour of the carotid artery plaque (CAP). In C, the patent lumen of the carotid is visible (L indicates lumen), whereas in $D$, the opacified patent lumen is also indicated (OL indicates opacified lumen). In the histologic section (E), the rupture of the FFC is noted (black arrows).

\section{MATERIALS AND METHODS}

\section{Study Design and Patient Population}

We obtained institutional review board approval for this study. Forty-seven consecutive symptomatic patients $($ men $=25$; women $=22$; mean age $=66.8 \pm 9$ years) were prospectively analyzed from May 2010 to March 2012. The mean time interval between CTA and the clinical episode (stroke or TIA) was 4 days (range, 1-12 days). We considered as an inclusion criterion that the carotid endarterectomy (CEA) was performed within 10 days after the CTA. A subgroup of the current population $(n=29)$ was included in a previous study. ${ }^{9}$

\section{CT Technique}

CT was performed with the use of a 16-row multidetector CT system (Brilliance, Philips, Best, The Netherlands) with a previously described protocol. ${ }^{9}$ Briefly, the basal scan is performed, followed by the angiographic phase in the caudocranial direction. The volume of injected contrast is $80 \mathrm{~mL}$ (Ultravist 370; Bayer Schering Pharma, Berlin, Germany), with a flow rate of $5 \mathrm{~mL} / \mathrm{s}$. The precise timing of the injection is obtained with a bolus-tracking technique: the dynamic monitoring began 6 seconds after the beginning of the intravenous injection of contrast material. The delay between the acquisitions of each monitoring image was 1 second. When the threshold was reached (by using a threshold inside the region of interest set +90 Hounsfield units [HU] above the baseline as a trigger), the patient was instructed not to breathe; after an interval of 4 seconds, the CTA acquisition started in the caudocranial direction from the aortic arch to the circle of Willis, with a section thickness of $1.3 \mathrm{~mm}$, increment $0.6 \mathrm{~mm}$, matrix $512 \times 512$, FOV $16-19 \mathrm{~cm}$, mAs $260-300$, and $\mathrm{kV}$ 120-140. An intermediate reconstruction algorithm (Cfilter) was used.

\section{Carotid Plaque Enhancement Quantification}

The CPE quantification was performed by use of a validated technique. ${ }^{9-11}$ Two radiologists performed all measurements of $\mathrm{HU}$ (L.S. and E.R.) by using as window/level settings W850:L300. ${ }^{12}$ Particular care was taken to obtain a correct registration between the basal phase and the enhanced phase (Fig 1A,-B). The HU quantification is obtained by use of a circular or elliptical region of interest $\left(\geq 1 \mathrm{~mm}^{2}\right)$.The threshold to consider the CPE as present was a variation of $\geq 10 \mathrm{HU}^{9}$

\section{Carotid Endarterectomy}

CEA was performed by 2 vascular surgeons (R.M. and Robert Sanfilippo) by use of the en bloc technique (to reduce the manipulation of the carotid plaque and avoid the potential rupture/fragmentation of the plaque), by scoring the adventitia and outer media with a scalpel and removing the plaque as an intact tube. The criteria to perform carotid endarterectomy were based on the recommendations by the 
NASCET and European Carotid Surgery Trial studies for symptomatic patients. $^{13-15}$

\section{Histologic Analysis of the Plaque}

Histologic examination was performed by 2 observers (L.S. and E.R.) blinded to clinical-radiologic data. The plaque specimen was immediately fixed in formalin, and it was taken to the laboratory directly after the surgical procedure. None of the carotid endarterectomy specimens showed disruption of the luminal surface of the plaque. Carotid plaque was decalcified and embedded in paraffin wax. The portion of the specimen showing the carotid plaque was divided transversely in sections at 3-mm intervals that were air-dried at $60^{\circ} \mathrm{C}$ for 45 minutes. After this phase, paraffin was removed by xylol and the sections were hydrated. Finally, endogenous peroxidase activity was blocked by $2 \% \mathrm{H}_{2} \mathrm{O}_{2}$. The 5 - $\mu \mathrm{m}$ transverse sections were subjected to histologic examination to identify the plaque components, the fibrous cap, and its rupture (Fig $1 C$ ). The fibrous cap components such as attenuated and loose (proteoglycan) matrix, hemorrhage, neovasculature, calcification, and inflammatory cell infiltrate were analyzed, and the condition of FFC was identified by the loss of anatomic continuity (integrity) of the fibrous cap. ${ }^{6}$ In addition, the neovascularization was assessed by analyzing the microvessel attenuation according to the methodology indicated by Saba et al. ${ }^{9}$

Table 1: Patient characteristics

\begin{tabular}{lccc}
\hline \multicolumn{1}{c}{ Parameter } & FFC & Non-FFC & P Value \\
\hline Patients, $n$ & $12(25.5 \%)$ & $35(74.5 \%)$ & NC \\
CPE, HU & $22.6 \pm 10.9$ & $12.9 \pm 9.4$ & .001 \\
CPE, 95\% Cl, HU & $12.8-33.1$ & $10.2-20.8$ & NC \\
Age, y & $67 \pm 8$ & $65 \pm 9$ & .495 \\
Sex, male & $8(67 \%)$ & $17(49 \%)$ & .278 \\
Smoker & $6(55 \%)$ & $19(54 \%)$ & .938 \\
Hypertension & $7(58 \%)$ & $22(63 \%)$ & .781 \\
CAD & $8(67 \%)$ & $19(54 \%)$ & .454 \\
Diabetes & $3(25 \%)$ & $3(9 \%)$ & .141 \\
Dyslipidemia & $7(58 \%)$ & $14(40 \%)$ & .271 \\
Statins and other drugs ${ }^{a}$ & $6(50 \%)$ & $14(40 \%)$ & .545 \\
\hline
\end{tabular}

Note:-CAD indicates coronary artery disease; NC, not calculable. a Other lipid-lowering drugs.

\section{Calculation of the Radiation Dose}

For each examination, the CT dose index (CTDI), dose-length product (DLP), and length of the scans in centimeters was collected. The effective dose (measured in $\mathrm{mSv}$ ) was also calculated by converting the DLP by use of the following conversion equation for CT of the neck: $\mathrm{mSv}=0.0059^{*}$ DLP. $^{16}$

\section{Statistical Analysis}

Continuous quantitative variables were expressed as mean \pm standard deviations. The plaque HU values were averaged between the 2 radiologists, and the Bland-Altman statistic was performed. The Mann-Whitney test was used to test the differences between the 2 groups of patients, with and without FFC, and the nonparametric McNemar test was used to test the difference between the prevalence of FFC in carotid arteries with and without CPE. Receiver operating characteristic (ROC) curve analysis was also performed to test the association between the presence of FFC and CPE; the area under the curve was also calculated. Multiple logistic regression analysis was also performed to assess the effect of the FFC versus the microvessel attenuation. R software (www.r-project.org) was used for statistical analyses.

\section{RESULTS}

\section{General Results}

Demographic and CPE characteristics of the studied patients are shown in Table 1. In the 47 carotid arteries studied, 12 cases with FFC were found. In $92 \%(11 / 12)$ of the plaques with FFC we found CPE, whereas in only $69 \%$ (24/35) of the plaques without FFC, was CPE found and the McNemar test confirmed that there was a statistically significant difference $(P=.0001)$ Bland-Altman analysis demonstrated very good concordance between readers, with a mean difference between them of $4 \%$.

\section{Mann-Whitney Test}

The Mann-Whitney test showed a statistically significant difference between the amount of CPE in plaques with $\mathrm{FFC}(\mathrm{HU}=22.6 \pm 10.9)$ and without FFC $(\mathrm{HU}=12.9 \pm 9.4)(P=.011)($ Fig $2 A)$.
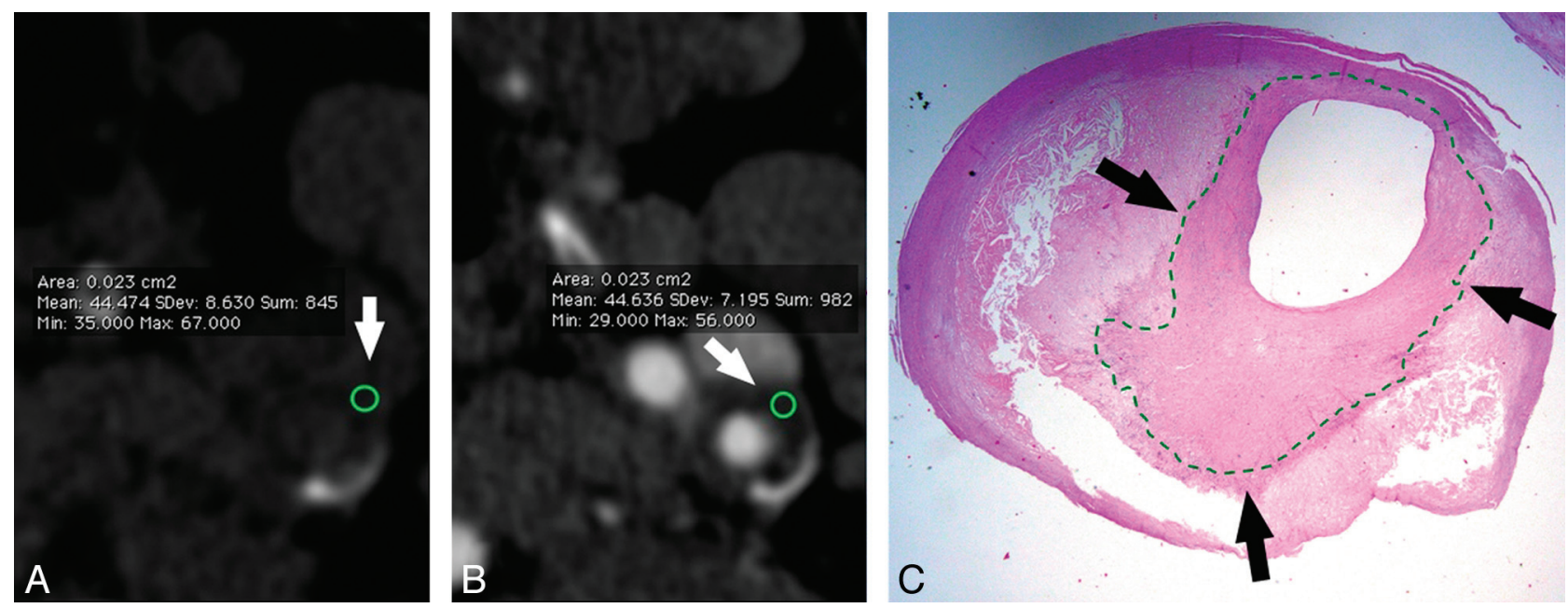

FIG 2. A 68-year-old male patient. In CT analysis, before $(A)$ and after (B) contrast, the carotid artery plaque is visible (white arrows) and the CPE $\mathrm{HU}$ value is shown. The circles, indicated by the white arrows, represent the area of the plaque that was assessed; in the basal scan, the average $\mathrm{HU}$ value was 44.474, whereas after administration of contrast material it was 44.636 . In this case, there was no contrast plaque enhancement. In the histologic section (C), the thick fibrous cap is visible (black arrows). 


\section{ROC Curve Analysis}

The ROC curve analysis (Fig 2B) confirmed an association between FFC and CPE, with an area under the curve of 0.749 (95\% confidence interval $=0.601-0.864 ; P=.005$; standard error $=$ 0.089).

\section{Multiple Logistic Regression Analysis}

The multiple logistic regression analysis was performed to assess the effect of the FFC on the CPE by avoiding the confounding effect of the neovascularization (Table 2). We found that both FFC and neovascularization had a statistically significant association with the presence of $\operatorname{CPE}(P=.0366$ and .0001 , respectively).

\section{Radiation Dose Analysis}

The radiation dose analysis was performed in our cohort of patients, and the results are summarized in Table 3.

\section{DISCUSSION}

CTA is widely used for the imaging of carotid arteries; this technique allows for the study of plaque morphology and for the quantification and characterization of plaque composition with excellent detail. In CTA, carotid artery plaques may dem-

Table 2: Multiple logistic regression analysis

\begin{tabular}{lccccc}
\hline $\begin{array}{c}\text { Independent } \\
\text { Variables }\end{array}$ & Coefficient & $\begin{array}{c}\text { Standard } \\
\text { Error }\end{array}$ & $\boldsymbol{t}$ & $\begin{array}{c}\boldsymbol{P} \\
\text { Value }\end{array}$ & $\boldsymbol{r}$ \\
\hline (Constant) & 0.23552 & & & & \\
FFC & 0.25401 & 0.1178 & 2.156 & .0366 & 0.208 \\
Neovascularization & 0.19714 & 0.04244 & 4.645 & $<.0001$ & 0.539 \\
\hline
\end{tabular}

onstrate the presence of contrast enhancement, ${ }^{9-11}$ which is associated with the presence of cerebrovascular symptoms ${ }^{10,11}$; the reasons underlying the enhancement are otherwise unclear.

In our study, we found that the plaques with FFC enhance more often than do the plaques without FFC: in $92 \%$ of the plaques with FFC we found CPE, whereas in only $69 \%$ of the plaques without FFC, was $\mathrm{CPE}$ found $(P=.0001)$. These results are also concordant with histologic and fluid-dynamic analyses that have clarified that the rupture of the FFC creates a breach into the lipid-rich necrotic core with blood flow that actively enters into the plaque by determining pro-thrombotic effects. ${ }^{17,18}$ In the only case of FFC without CPE, the histologic sections were reanalyzed and an absence of lipid-rich necrotic core was detected. This fact suggests to us that the configuration of the core of the plaque may play a role: in these cases with a weak necrotic core, the rupture of the plaque may be associated with a blood invasion into the plaque, whereas in the case of more robust configuration, the rupture of the fibrous cap cannot be associated with intraplaque hemorrhage.

Moreover, we also found that the amount of CPE is larger in the plaques with FFC (22.6 versus $12.9 ; P=.011)$. This can be explained by the fact that the rupture of the fibrous cap allows the blood to massively enter into the plaque. ROC curve analysis confirmed an association between FFC and CPE (Fig 3). These results suggest that FFC is one of the factors that determine the enhancement of the plaque. It was demonstrated that the neovascularization of the plaque is associated with the presence of $\mathrm{CPE}$, and this parameter may represent a confounding factor in the assessment of the effect of the FFC in CPE. Therefore, a multiple logistic

Table 3: Radiation dose parameters of the population for the basal or contrast-enhanced phase

\begin{tabular}{|c|c|c|c|c|c|c|c|}
\hline & $n$ & Mean & $95 \% \mathrm{Cl}$ & SD & Minimum & Maximum & 2.5-97.5 Percentiles \\
\hline CTDI, mGy & 47 & 11.657 & $11.510-11.805$ & 0.5021 & 10.9 & 13 & $11.035-12.933$ \\
\hline $\mathrm{DLP}, \mathrm{mG}{ }^{*} \mathrm{~cm}$ & 47 & 348.113 & $341.502-354.725$ & 22.5172 & 306.23 & 415 & $307.425-400.353$ \\
\hline Effective dose, mSv & 47 & 2.054 & $2.015-2.093$ & 0.1329 & 1.807 & 2.449 & $1.814-2.362$ \\
\hline Length & 47 & 29.866 & $29.416-30.316$ & 1.5339 & 27.1 & 34.2 & $27.370-33.525$ \\
\hline
\end{tabular}

Box Plot

Contrast Plaque Enhancement (HU)

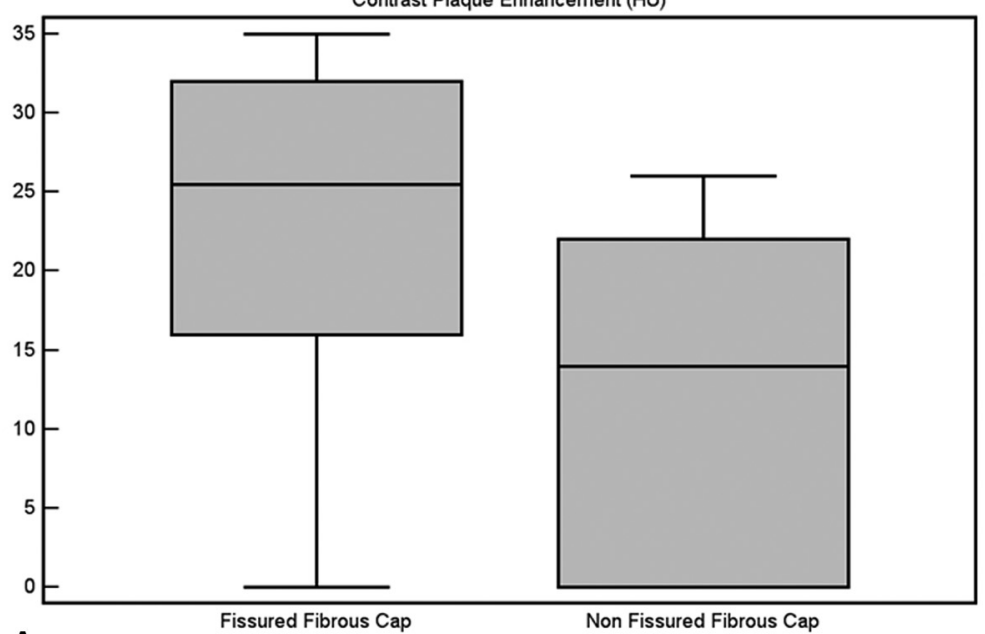

A

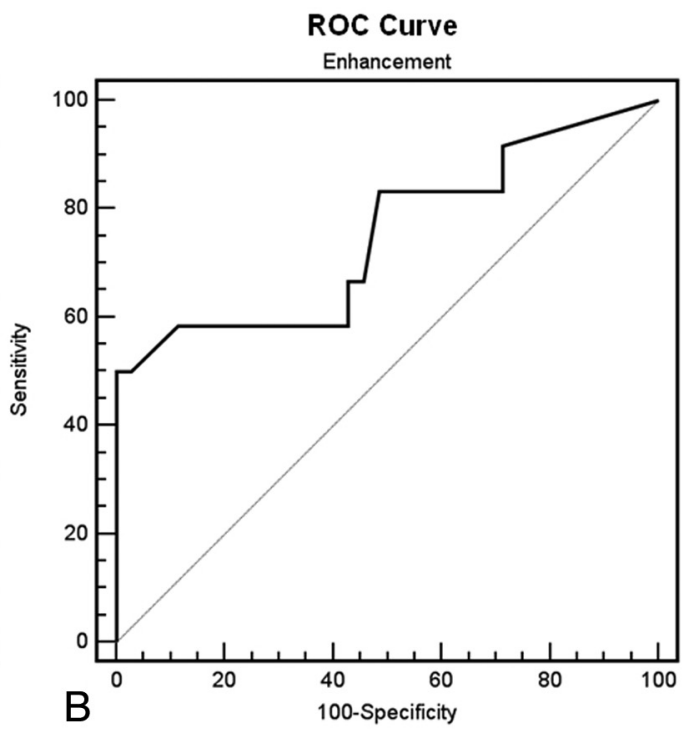

ROC Curve

FIG 3. Boxplot analysis shows the difference in CPE between the group of patients with FFC and the patients without FFC (A); the ROC curve shows the association between FFC and CPE (B). 
regression analysis was performed, and this confirmed that both FFC and neovascularization had a statistically significant association with the presence of CPE ( $P=.0366$ and .0001 , respectively $)$ and that they independently can determine the presence of CPE. These results may explain why in previous analysis, ${ }^{9}$ plaques with low levels of neovascularization had a large CPE. In our study, notably, the 2 groups of patients, with and without rupture of the FFC, were similar with regard to risk factors for carotid artery disease (Table 1). A similar result has been described with the use of MR imaging, ${ }^{7}$ but the description of this finding by use of CT has obvious consequences on the usefulness of this evaluation in clinical practice. The results are also concordant with histologic and fluid-dynamic analyses that have clarified that the rupture of the FFC creates a breach into the lipid-rich necrotic core with the blood flow that actively enters into the plaque by determining pro-thrombotic effects. ${ }^{17,18}$

In the past years, several investigations have demonstrated the potentialy of MR in the assessment of FFC status. ${ }^{1,2,6,7}$ However in several institutions, CTA is widely used in the assessment of the carotid artery status, in particular in emergency settings ${ }^{19}$ or for those subjects with contraindications to MR imaging such as claustrophobic patients or patients with pacemakers. Some advantages of CT are the short time of the procedure (the CT acquisition requires only few seconds) and the excellent spatial resolution that can reach $0.3 \mathrm{~mm}$ isotropic voxels. In recent years, several investigations have demonstrated the potential of CT in the assessment of carotid artery plaque composition ${ }^{20,21}$ by suggesting that some parameters, easily detectable with CT, are important for stroke risk stratification. With the current study, we showed that the presence of FFC is associated with contrast plaque enhancement and that this should be considered a further useful element for the stratification of stroke risk. It is not our opinion that the CTA should be performed to merely test the presence or absence of the CPE; once the CTA is performed, we think that this element should be assessed.

The 2 main disadvantages of CTA are the nephrotoxicity and radiation dose. To reduce the nephrotoxicity, we are currently using the "bolus technique," with a reduced volume of contrast material $(80 \mathrm{~mL})$ injected at a high flow rate. In the future, it is likely that the volume of the injected contrast material will be further reduced, thanks to the potential and velocity of the newer CT scanners. The radiation dose delivered to the patient is the second main issue related to the use of CT. In our cohort of patients, we had a mean CTDI of $11.657 \mathrm{mGy}$, which is a value similar to another recent publication where the single-source CT was used ${ }^{22}(12.5 \mathrm{mGy})$; the effective dose for each acquisition is similar. It is important to underline that there is an evolution with the new CT scanners, in particular the dualenergy systems that allow performing examinations with similar image quality and lower radiation dose compared with single-source CT. ${ }^{22}$

In this study, there are some limitations: the first is the number of analyzed patients $(n=47)$, which does not allow us to obtain a strong statistical analysis with tight confidence intervals; for this reason, the current results should be considered as preliminary results that must be confirmed in a larger population. The second limitation of this study is the potential plaque rupture during the surgeon's manipulation of the carotid arteries while performing the carotid endarterectomy. However, it is our hypothesis that this can be considered as a minor limitation because our surgeons used particular care in the CEA procedure; to reduce this effect, an ex vivo CEA should be performed.

\section{CONCLUSIONS}

The results of this preliminary study indicated that the presence of FFC is associated with CPE. Histologic analysis showed that the presence of FFC is associated with a larger $\mathrm{CPE}$ compared with the $\mathrm{CPE}$ of plaques without FFC.

\section{REFERENCES}

1. Hatsukami TS, Yuan C. MRI in the early identification and classification of high-risk atherosclerotic carotid plaques. Imaging Med 2010;2:63-75

2. Yuan C, Zhang SX, Polissar NL, et al. Identification of fibrous cap rupture with magnetic resonance imaging is highly associated with recent transient ischemic attack or stroke. Circulation 2002;105: $181-85$

3. Takaya N, Yuan C, Chu B, et al. Association between carotid plaque characteristics and subsequent ischemic cerebrovascular events: a prospective assessment with MRI: initial results. Stroke 2006; $37: 818-23$

4. Saba L, Potters F, van der Lugt A, et al. Imaging of the fibrous cap in atherosclerotic carotid plaque. Cardiovasc Intervent Radiol 2010;33:681-89

5. Watanabe $Y$, Nagayama M. MR plaque imaging of the carotid artery. Neuroradiology 2010;52:253-74

6. Hatsukami TS, Ross R, Polissar NL, et al. Visualization of fibrous cap thickness and rupture in human atherosclerotic carotid plaque in vivo with high-resolution magnetic resonance imaging. Circulation 2000;102:959-64

7. Cai J, Hatsukami TS, Ferguson MS, et al. In vivo quantitative measurement of intact fibrous cap and lipid-rich necrotic core size in atherosclerotic carotid plaque: comparison of high-resolution, contrast-enhanced magnetic resonance imaging and histology. Circulation 2005;112:3437-44

8. Saba L, Mallarini G. Fissured fibrous cap of vulnerable carotid plaques and symptomaticity: are they correlated? Preliminary results by using multi-detector-row CT angiography. Cerebrovasc Dis 2009;27:322-27

9. Saba L, Lai ML, Montisci R, et al. Association between carotid plaque enhancement shown by multidetector CT angiography and histologically validated microvessel density. Eur Radiol 2012;22:2237-45

10. Saba L, Piga M, Raz E, et al. Carotid artery plaque classification: does contrast enhancement play a significant role? AJNR Am J Neuroradiol 2012;33:1814-17

11. Saba L, Mallarini G. Carotid plaque enhancement and symptom correlations: an evaluation by using multidetector row CT angiography. AJNR Am J Neuroradiol 2011;32:1919-25

12. Saba L, Mallarini G. MDCTA in the study of carotid plaque stenosis degree: evaluation of inter-observer agreement. AJR Am J Roentgenol 2008; 190:W41-46

13. North American Symptomatic Carotid Endarterectomy Trial Collaborators. Beneficial effect of carotid endarterectomy in symptomatic patients with high-grade stenosis. N Engl J Med 1991;325:445-53

14. Barnett HJ, Taylor DW, Eliasziw M, et al. Benefit of carotid endarterectomy in patients with symptomatic moderate or severe stenosis: North American Symptomatic Carotid Endarterectomy Trial Collaborators. N Engl J Med 1998;339:1415-25

15. Rothwell PM, Eliasziw M, Gutnikov SA. Analysis of pooled data from the randomised controlled trials of endarterectomy for symptomatic carotid stenosis. Lancet 2003;361:107-16

16. Bongartz G, Golding SJ, Jurik AG. CT quality criteria. Luxembourg: European Commission; 2004 
17. Redgrave JN, Gallagher P, Lovett JK, et al. Critical cap thickness and rupture in symptomatic carotid plaques: the Oxford Plaque Study. Stroke 2008;39:1722-9

18. Cicha I, Wörner A, Urschel K, et al. Carotid plaque vulnerability: a positive feedback between hemodynamic and biochemical mechanisms. Stroke 2011;42:3502-10

19. Magge R, Lau BC, Soares BP, et al. Clinical risk factors and CT imaging features of carotid atherosclerotic plaques as predictors of new incident carotid ischemic stroke: a retrospective cohort study. AJNR Am J Neuroradiol 2013;34:402-09
20. Saba L, Sanfilippo R, Sannia S, et al. Association between carotid artery plaque volume, composition, and ulceration: a retrospective assessment with MDCT. AJR Am J Roentgenol 2012;199:151-56

21. van Gils MJ, Vukadinovic D, van Dijk AC, et al. Carotid atherosclerotic plaque progression and change in plaque composition over time: a 5-year follow-up study using serial CT angiography. AJNR Am J Neuroradiol 2012;33:1267-73

22. Paul J, Mbalisike EC, Nour-Eldin NE, et al. Dual-source 128-slice MDCT neck: radiation dose and image quality estimation of three different protocols. Eur J Radiol 2013;82:787-96 